\title{
M-LEARNING E MATEMÁTICA: MAPEANDO RECURSOS E MODALIDADES EDUCACIONAIS
}

\author{
Silvia Cristina Freitas Batista, PGIE/UFRGS, IF Fluminense Campus Campos-Centro \\ silviac@iff.edu.br \\ Patricia Alejandra Behar, NUTED-PGIE/UFRGS \\ patricia.behar@ufrgs.br
}

\section{RESUMO}

A popularização das Tecnologias Móveis Sem Fio e a importância da mobilidade e da interatividade têm feito da m-learning (mobile learning - aprendizagem por meio de dispositivos móveis) um interessante campo de investigação educacional. Este artigo focaliza uma área específica de aplicação de m-learning, a aprendizagem matemática. Neste sentido, analisam-se estudos sobre a utilização de m-learning na referida área e descrevem-se recursos específicos para a mesma. Além disso, discute-se o campo de aplicação de m-learning que, além da educação a distância, vem abrangendo outras modalidades educacionais (presencial e semipresencial). Finalizando, descreve-se uma pesquisa exploratória, realizada no IF Fluminense, visando identificar indicativos sobre possibilidades de uso de dispositivos móveis, em cursos presenciais da referida instituição. De modo geral, os dados levantados indicaram a existência de um contexto receptivo a ações educacionais utilizando dispositivos móveis, em particular, celulares.

Palavras-chave: M-learning, Aprendizagem Matemática, Modalidades Educacionais.

\section{M-LEARNING AND MATHEMATICS: MAPPING RESOURCES AND EDUCATIONAL MODALITIES}

In today's society, the mobile wireless technologies are becoming increasingly popular. This fact, coupled with importance of mobility and interactivity, makes the m-learning (mobile learning - learning through mobile devices) an interesting field of educational research. This article focuses on a specific area of application of m-learning, learning mathematics. In this sense, we examine studies on the use of m-learning in that area and describe specific resources for the same. Furthermore, we discuss the scope of $\mathrm{m}$ learning that, in addition to distance education, has been covering other educational modalities (face-to-face and blended learning). Finally, we describe an exploratory research carried out at IF Fluminense, to identify indicative of potential uses of mobile devices, in courses face-to-face of this institution. Overall, the data obtained indicated the existence of a context receptive to educational activities using mobile devices, particularly cell phones.

Keywords: M-learning, Learning Mathematics, Educational Modalities.

\section{Introdução}

Tendências sociais têm indicado a formação de uma sociedade que pode ser denominada Sociedade da Comunicação Móvel, como parte da estrutura social mais ampla que é a Sociedade em Rede. Tempo e espaço são aspectos que expressam mais diretamente esta mudança social. Avanços tecnológicos, principalmente os relacionados à comunicação, têm tido um impacto crítico sobre essa mudança espaço-temporal, o que não significa, certamente, que a tecnologia aja de forma isolada de outras fontes mais amplas de mudança (Castells et al., 2004). 
Nesse cenário, as Tecnologias Móveis Sem Fio têm despertado grande interesse em termos educacionais. M-learning (mobile learning - aprendizagem por meio de dispositivos móveis) é um campo emergente, que engloba tecnologias sem fio e computação móvel para permitir que a aprendizagem possa ocorrer em qualquer tempo e em qualquer lugar, maximizando a liberdade dos alunos (Wains; Mahmood, 2008).

Aplicações simples de m-learning podem ser citadas por meio do uso de SMS (Short Messaging System), tais como comunicações acadêmicas e testes de múltipla escolha (Keegan, 2007). No entanto, há formas mais complexas de aplicação e, nesse sentido, diversos estudos relacionados a ambientes de aprendizagem para dispositivos móveis têm sido realizados (Meisenberger, 2004; Scopel et al., 2004; Meirelles e Tarouco, 2005; Brown et al., 2006; Sá et al., 2009). Além disso, vários estudos sobre desenvolvimento de materiais didáticos para m-learning têm sido promovidos (Tarouco et al., 2004; Dye, 2007; Costello, 2007; Costabile, 2008).

Em particular, pesquisas têm buscado verificar como m-learning pode ser útil ao processo de ensino e aprendizagem de Matemática (Botzer e Yerushalmy, 2007; Franklin e Peng, 2008; Nokia, 2009; Baya'a e Daher, 2009). De maneira geral, as pesquisas mencionadas apontam diversas vantagens do uso de dispositivos móveis para aprendizagem matemática, tais como: i) visualização e investigação dinâmica de fatos matemáticos; ii) formas diferentes de abordagem de conceitos (por exemplo, por meio de vídeos, trabalhando abordagens visuais); iii) autonomia no estudo de temas matemáticos; iv) aprendizagem em situações reais.

Nesse contexto, este artigo analisa estudos e recursos relacionados à utilização de m-learning na aprendizagem matemática e discute as modalidades educacionais (educação presencial, semipresencial e a distância) que podem se beneficiar dessa forma de aprendizagem. Além disso, tendo em vista ações futuras a serem desenvolvidas no IF Fluminense, envolvendo m-learning e Matemática, analisam-se dados coletados em uma pesquisa exploratória. A referida pesquisa teve por objetivo identificar indicativos sobre possibilidades de uso de dispositivos móveis, em cursos presenciais da referida instituição. Para tanto, na seção 2 são descritos alguns projetos e recursos de m-learning direcionados à Matemática. Na seção 3, analisa-se o campo de aplicação de m-learning, que tem se ampliado para outras modalidades educacionais, além da educação a distância. Na seção 4, são analisados dados da pesquisa exploratória, promovida em três cursos presenciais do IF Fluminense Campus Campos-Centro. Finalizando, a seção 5 apresenta algumas considerações sobre o tema abordado.

\section{M-learning e Matemática: pesquisas e recursos}

Nesta seção, apresentam-se, brevemente, pesquisas sobre o uso de m-learning na aprendizagem matemática. Além disso, são descritos alguns recursos específicos para a referida área, em celulares.

Em outubro de 2008, a Nokia e a Nokia Siemens Networks iniciaram um projeto ${ }^{1}$ de m-learning para Matemática, na África do Sul, relacionado à aprendizagem formal. O projeto contou com a colaboração do governo do referido país e foi implementado no período de fevereiro a maio de 2009. Tendo por foco uma aprendizagem ativa, os materiais educacionais eram enviados para os celulares dos alunos por meio de um canal colaborativo de entrega (o MXit, um serviço de mensagens instantâneas, via Internet, sem usar a tecnologia padrão de SMS). O conteúdo incluía exercícios, passo-apasso de resoluções, sugestões e dicas áudio-visuais para ajudar nas resoluções (Nokia, 2009). Os alunos podiam encaminhar resultados de trabalhos, bem como receber feedback e lembretes dos professores, via SMS. Eventuais problemas podiam ser discutidos com os professores, em tempo real, usando Dr Maths, um aplicativo online 
para tutoria (Nokia, 2009). Os primeiros resultados mostraram que, na visão dos alunos, as tecnologias adotadas, realmente, ajudaram nas aulas de Matemática. A utilização de m-learning também colaborou para que os professores conhecessem de forma mais ampla as competências dos alunos, assim como possibilitou que os próprios alunos compreendessem melhor suas capacidades (Nokia, 2009).

Franklin e Peng (2008) também descrevem uma experiência que consideraram válida para a aprendizagem matemática e para a prática de trabalho em equipe. A referida experiência foi um estudo de caso no qual o iPod Touch® foi utilizado em duas turmas de uma escola dos EUA (8th Grade - Middle School). Os alunos elaboraram vídeos sobre tópicos matemáticos, utilizando o referido dispositivo. Segundo os autores, a habilidade dos alunos para apresentar conceitos complexos por meio visual (nos vídeos) e, depois, discuti-los com os colegas, foi impressionante. A preocupação dos professores, no início do estudo, relacionada a possíveis dificuldades dos alunos em utilizar as tecnologias e em desenvolver os vídeos, mostrou-se infundada, pois, com algumas orientações, os alunos trabalharam sem demonstrar problemas. O trabalho realizado foi diferente para todos, tanto professores quanto alunos. Foi preciso adaptar o mundo dos números ao modelo visual de representação, o que, na verdade, segundo os autores, é um conceito novo (abordagem visual) também para a maioria dos professores de Matemática.

Outro estudo de caso é relatado por Baya'a e Daher (2009). A experiência ocorreu por meio de uma atividade extraclasse, com estudantes de uma escola de Israel (8th grade - Middle School). Os alunos utilizaram aplicativos gráficos para celulares, específicos para Matemática (trabalhando com funções lineares), e também recursos dos próprios celulares, como fotografias, vídeos, entre outros. Segundo os autores, os alunos ficaram, positivamente, impressionados com as potencialidades dos recursos utilizados, o que sinalizaria que estes podem contribuir para a aprendizagem matemática. Dentre as vantagens apresentadas pelos alunos, destacam-se: i) autonomia na exploração dos temas matemáticos; ii) aprendizagem por meio de colaboração e trabalho em grupo; iii) aprendizagem em contexto real; iv) visualização e investigação dinâmica de fatos matemáticos; v) aprendizagem da matemática com facilidade e eficiência (Baya'a; Daher, 2009). Os aplicativos gráficos para celulares, utilizados nesse estudo de caso, são do Projeto Math4Mobile.

O Math4Mobile é um projeto do Instituto para Alternativas em Educação, vinculado à Universidade de Haifa, Israel. Tendo como termos-chave mobilidade, disponibilidade e flexibilidade, o referido projeto visa aproveitar as oportunidades oferecidas pelas tecnologias móveis, particularmente celulares, em benefício da aprendizagem matemática (Math4Mobile, s.d.). No site do Math4Mobile (http://www.math4mobile.com/) estão disponíveis, para download, cinco aplicativos gratuitos (para uso não comercial), em Java (J2ME), destinados ao estudo investigativo de diversos conceitos matemáticos, por meio do celular. São eles:

- Graph2Go (Figura 1a) - opera como uma calculadora gráfica, para um dado conjunto de funções, permitindo estabelecer conexões entre representações gráficas e algébricas, por meio de transformações dinâmicas. Utilizando as setas Direita/Esquerda do teclado do celular, é possível selecionar o coeficiente da função a ser alterado. Com as setas Para Cima/Para Baixo, aumenta-se e diminui-se, respectivamente, o valor do coeficiente escolhido;

- Quad2Go (Figura 1b) - possibilita o estudo de quadriláteros, permitindo levantar conjecturas por meio dos inúmeros exemplos que podem ser obtidos pela movimentação de elementos. Explorações com Quad2Go são, especialmente, apropriadas para estudantes de 11-12 anos de idade;

V. $7 \mathrm{~N}^{\mathrm{o}}$ 3, dezembro, 2009 
- Solve2Go - possibilita a resolução de equações e inequações, com base em análises gráficas;

- Sketch2Go - é uma ferramenta gráfica qualitativa. Os gráficos são traçados utilizando ícones que indicam funções constantes, crescentes e decrescentes, que variam segundo taxas (derivadas) constantes, crescentes ou decrescentes;

- Fit2Go - possibilita a exploração e modelagem de atividades, recebendo dados coletados e propondo um modelo (em termos de função linear ou quadrática) que possa melhor descrevê-los. Juntos, Sketch2Go e Fit2Go podem fornecer uma visão ampla de modelos e modelagem.

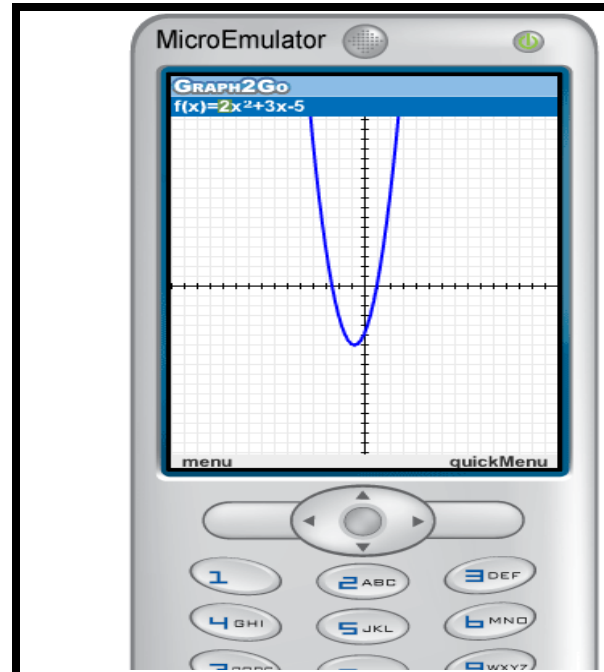

Figura 1a: Graph2Go

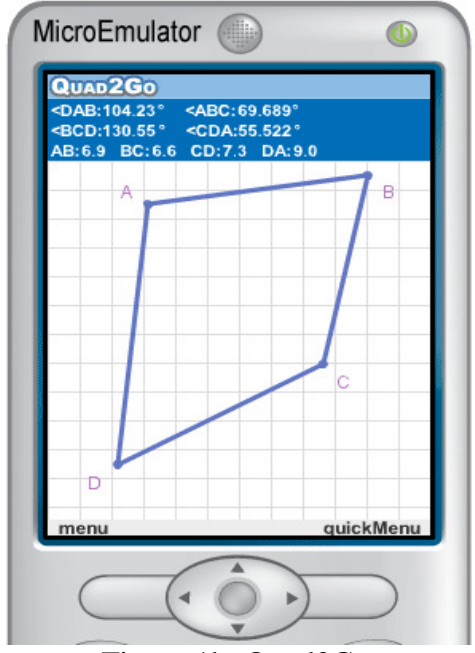

Figura 1b: Quad2Go

Figura 1: Aplicativos do Projeto Math4Mobile

Observa-se que os estudos e recursos, descritos nesta seção, não focam a educação a distância. Na literatura da área, no entanto, é comum encontrar associações entre mleaning e e-learning (Quinn, 2000; Georgiev et al., 2004; Wains e Mahmood, 2008), sendo e-learning uma forma de educação a distância, baseada em computador e Internet. $\mathrm{Na}$ seção seguinte discutem-se as modalidades educacionais nas quais o uso de mlearning vem sendo pesquisado.

\section{M-learning em diversas modalidades educacionais}

Diversos estudos associam m-learning à e-learning. Quinn (2000) defende que mlearning é e-learning desenvolvida por meio de dispositivos móveis. Reiterando essa visão, Georgiev et al. (2004), afirmam que a m-learning pode ser entendida como um novo estágio da educação a distância (d-learning) e da e-learning, como mostra a Figura 2. Wains e Mahmood (2008) afirmam que m-learning visa atender alguns aspectos que ainda prejudicam a e-learning, como falta de infra-estrutura de acesso à Internet nos países em desenvolvimento e a questão da mobilidade dos alunos. 


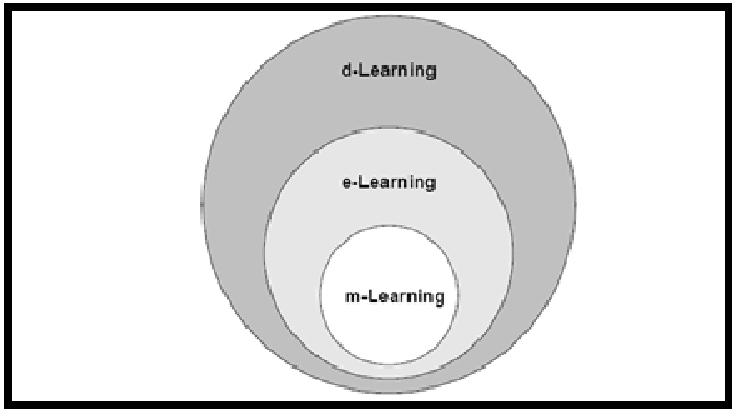

Figura 2: M-learning em e-learning (Fonte: Georgiev et al., 2004)

No entanto, alguns estudos têm associado m-learning também à educação presencial e semipresencial (Franklin e Peng, 2008; Nokia, 2009; Khaddage e Lattemann, 2009; Zeiller, 2009). Segundo Zeiller (2009), a educação semipresencial pode utilizar tanto recursos da Web quanto dispositivos móveis na comunicação entre alunos e professores e nos trabalhos colaborativos. Khaddage e Lattemann (2009) defendem que é possível tirar proveito dos dispositivos móveis, no contexto de sala de aula, favorecendo aspectos como acessibilidade, colaboração e flexibilidade.

Dessa forma, a figura 2, proposta por Georgiev et al. (2004) não mais reproduziria o contexto real de m-learning. Considerando que pesquisas têm sinalizado que as potencialidades de m-learning podem colaborar, também, na educação presencial e semipresencial, a presente abordagem propõe uma redefinição da figura 2 , como ilustrado na figura 3. Nesta, as setas, em sentido duplo, entre m-learning e as modalidades educacionais, indicam que não só estas podem se beneficiar da m-learning, como também a m-learning pode evoluir com as experiências realizadas. Estudos envolvendo m-learning colaboram para o próprio desenvolvimento desta área, seja pela identificação de novas formas de aplicação, limitações e vantagens, ou pela ampliação e melhoria de recursos.

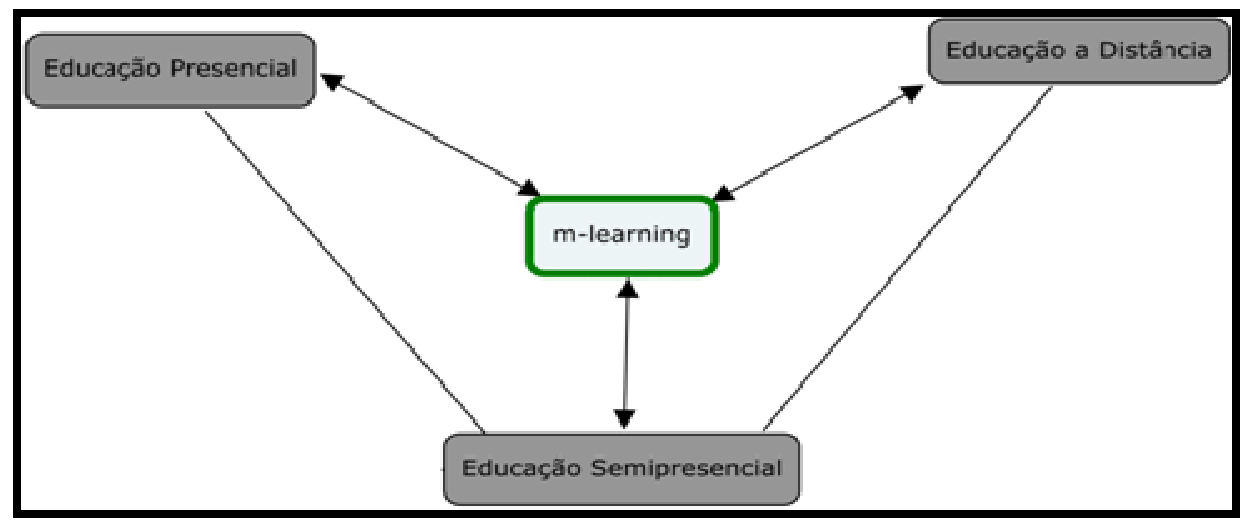

Figura 3: M-learning em várias modalidades educacionais

No contexto da figura 3, as modalidades educacionais são definidas como: i) Educação Presencial - modalidade "dos cursos regulares, em qualquer nível, onde professores e alunos se encontram sempre num local físico, chamado sala de aula" (Moran, p.1, 2002); ii) Educação Semipresencial - ocorre parte na sala de aula e parte a distância, por meio de tecnologias (Moran, 2002); iii) Educação a Distância - “[...] modalidade educacional, na qual a mediação didático-pedagógica nos processos de ensino-aprendizagem ocorre com a utilização de meios e tecnologias de informação e comunicação, com estudantes e professores desenvolvendo atividades educativas em lugares ou tempos diversos" (Brasil, 2005, p.1).

Quanto à Matemática, em particular, considera-se que esta precisa buscar formas de V. $7 \mathrm{~N}^{\mathrm{o}}$ 3, dezembro, 2009 
se tornar mais próxima do aluno. Muitas vezes, a Matemática desempenha um papel decisivo na vida das pessoas, rotulando-as e posicionando-as como aptas ou inaptas à participação nos processos de decisão da sociedade (Matos, 2005). Nesse sentido, considerando-se as justificativas para a figura 3 e os estudos descritos na seção 2, que sinalizam que m-learning tem potencialidades para contribuir para a aprendizagem matemática presencial, propõe-se a figura 4 (uma adequação da figura 3 ao contexto da Matemática).

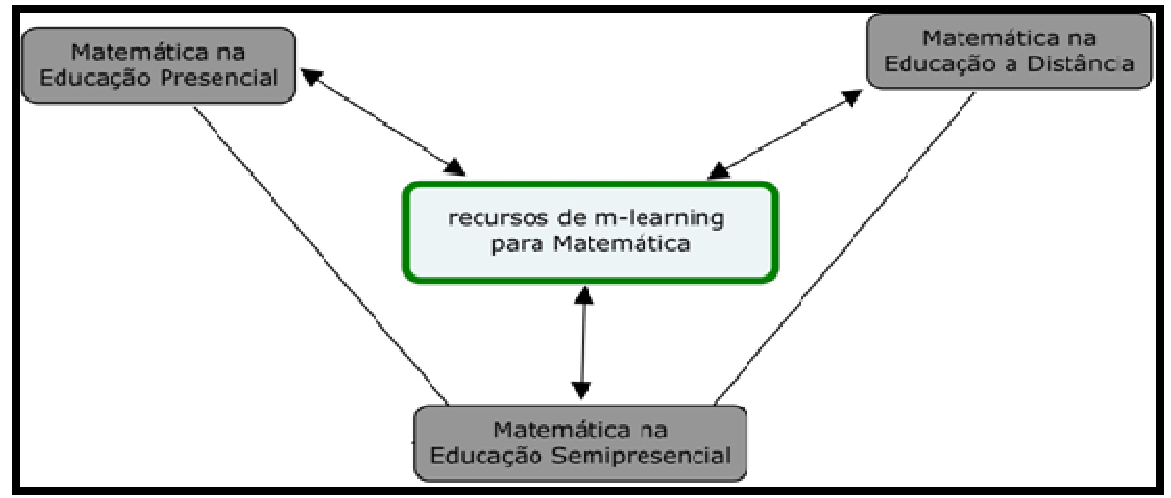

Figura 4: M-learning na aprendizagem matemática - várias modalidades educacionais

Entende-se que estudos devem contemplar as três modalidades (Figura 4), visando analisar a importância da m-learning para o processo de ensino e aprendizagem de Matemática.

Nesse sentido, tendo em vista ações futuras relacionadas a m-learning na aprendizagem matemática, foi realizada uma pesquisa que buscou identificar indicativos sobre possibilidades de uso de dispositivos móveis, em três cursos presenciais do IF Fluminense Campus Campos-Centro. Os dados levantados na referida pesquisa são analisados na seção seguinte.

\section{Mapeamento de perfil de usuários: levantando indicativos}

Em um primeiro momento, foi realizada uma pesquisa exploratória, que buscou levantar indicativos sobre possibilidades de uso de dispositivos móveis, em três cursos do IF Fluminense Campus Campos-Centro. Os referidos cursos foram Tecnologia em Análise e Desenvolvimento de Sistemas, Licenciatura em Matemática e Engenharia de Controle e Automação Industrial. Uma pesquisa exploratória visa, em geral, responder à necessidade de compreensão de certos aspectos da natureza do problema (Hernandez Sampieri et al., 1998).

Foram distribuídos, aleatoriamente, 20 questionários em cada um dos referidos cursos (não houve seleção de alunos por períodos letivos, o único critério para a distribuição do questionário foi freqüentar um dos três cursos).

A média de idade dos participantes da Licenciatura em Matemática foi de, aproximadamente, 21 anos, sendo 17 pessoas do sexo feminino e 3 do masculino. Na Engenharia, a média de idade dos participantes foi de, aproximadamente, 20 anos, sendo 15 pessoas do sexo masculino e 5 do feminino. Já no curso de Análise e Desenvolvimento de Sistemas, a média de idade dos participantes foi de 24 anos, sendo 13 pessoas do sexo masculino e 7 do feminino. O questionário utilizado na pesquisa, continha perguntas sobre dados pessoais (idade, sexo), dados do curso (nome do curso, período) e oito perguntas sobre dispositivos móveis (duas fechadas e seis semi-abertas, com opção "outros" ou solicitação de comentários).

Destacam-se aqui apenas dados considerados mais significativos para o contexto deste artigo. Ressalta-se que todos os participantes afirmaram possuir celular (comum, 
smartphone ou aparelhos MP10 ou MP11), com uma significativa predominância do celular comum sobre os demais tipos. Foi possível observar que os demais dispositivos móveis (PDAs, Palmtops, netbooks, entre outros) são pouco comuns entre os participantes. $\mathrm{O}$ quadro 1 mostra os percentuais relacionados à habilidade em lidar com o teclado do celular.

Quadro1: Habilidade em usar o teclado do celular

\begin{tabular}{|c|c|c|c|}
\hline \multirow{2}{*}{$\begin{array}{c}\text { Habilidade em usar o } \\
\text { teclado do celular }\end{array}$} & $\begin{array}{c}\text { Porcentagem de participantes de cada curso por categoria } \\
\text { Licenciatura em } \\
\text { Matemática }\end{array}$ & $\begin{array}{c}\text { Engenharia de } \\
\text { Controle e } \\
\text { Automação }\end{array}$ & $\begin{array}{c}\text { Análise e } \\
\text { Desenvolvimento de } \\
\text { Sistemas }\end{array}$ \\
\hline Péssima & - & $5 \%$ & - \\
\hline Ruim & $10 \%$ & $5 \%$ & - \\
\hline Regular & 0 & $10 \%$ & $15 \%$ \\
\hline Boa & $70 \%$ & $60 \%$ & $55 \%$ \\
\hline Excelente & $20 \%$ & $20 \%$ & $30 \%$ \\
\hline
\end{tabular}

Neste quadro, assim como nos demais, $100 \%$ dos participantes, em cada curso, são correspondentes a 20 alunos. De modo geral, são muito favoráveis, em termos educacionais, os percentuais obtidos. Considerando-se, conjuntamente, as categorias "Boa" e "Excelente", tem-se 80\% (Engenharia) como percentual mais baixo. Quanto à Licenciatura, estes percentuais se tornam, particularmente, interessantes, pois essa habilidade com o teclado pode ser bem aproveitada para m-learning, em práticas profissionais futuras destes alunos, se houver preparação nesse sentido.

O quadro 2 apresenta os percentuais relacionados aos custos de utilização do celular. Essa questão visou levantar indicativos sobre a influência que os custos podem ter sobre a utilização de certos serviços, em termos educacionais.

Quadro2: Custos de utilização como limitador de uso

\begin{tabular}{|c|c|c|c|}
\hline \multirow{2}{*}{$\begin{array}{c}\text { De maneira geral, os } \\
\text { custos de utilização são, } \\
\text { ainda, fatores } \\
\text { limitadores de uso? }\end{array}$} & $\begin{array}{c}\text { Porcentagem de participantes de cada curso por categoria } \\
\text { Licenciatura em } \\
\text { Matemática }\end{array}$ & $\begin{array}{c}\text { Engenharia de } \\
\text { Controle e } \\
\text { Automação }\end{array}$ & $\begin{array}{c}\text { Análise e } \\
\text { Desenvolvimento de } \\
\text { Sistemas }\end{array}$ \\
\hline Sim & $45 \%$ & $30 \%$ & $45 \%$ \\
\hline Parcialmente & $50 \%$ & $40 \%$ & $45 \%$ \\
\hline Não & $5 \%$ & $30 \%$ & $10 \%$ \\
\hline
\end{tabular}

Considerando-se, conjuntamente, no quadro 2, as categorias "Sim" e "Parcialmente", tem-se como percentual mais baixo 70\% (Engenharia), o que sinaliza o custo como um fator que, ainda, influencia, significativamente, o uso de recursos do celular. Nessa questão foi solicitado um comentário relacionado à opção escolhida. A maioria dos comentários abordou o fator custo de conexão à Internet. Apresentam-se três destes comentários:

(Sim) As mensagens com imagens são muito caras, e a navegação na internet também, caso fosse mais acessível usaria com maior freqüência (Aluno 6 Licenciatura).

(Parcialmente) Somente para o uso da internet (Aluno 5 - Tecnólogo).

(Não) Como o meu celular (MP11) possui internet wi-fi, em muitos lugares eu acesso a internet de graça. (Aluno 17 - Engenharia). 
Observa-se, no comentário do Aluno 17 da Engenharia, que, embora ele tenha acesso gratuito à Internet, a mesma está restrita a certos locais, o que é um aspecto que, de certa forma, entra em conflito com a questão da mobilidade.

O quadro 3 apresenta os percentuais sobre o uso de dispositivos móveis na educação. Percebe-se uma postura bastante receptiva à idéia do uso dos dispositivos móveis em termos educacionais. Os que responderam afirmativamente, de maneira geral, destacaram a importância do uso de tecnologias digitais na educação, a praticidade e mobilidade permitida pelos dispositivos móveis.

Quadro3: Dispositivos móveis na educação

\begin{tabular}{|c|c|c|c|}
\hline \multirow{2}{*}{$\begin{array}{l}\text { O uso de aparelhos móveis, com } \\
\text { conexão Internet, (sem ser } \\
\text { netbooks ou notebooks) para } \\
\text { fins educacionais é viável? }\end{array}$} & \multicolumn{3}{|c|}{ Porcentagem de participantes de cada curso por categoria } \\
\hline & $\begin{array}{c}\text { Licenciatura em } \\
\text { Matemática }\end{array}$ & $\begin{array}{l}\text { Engenharia de } \\
\text { Controle e } \\
\text { Automação } \\
\end{array}$ & $\begin{array}{c}\text { Análise e } \\
\text { Desenvolvimento de } \\
\text { Sistemas } \\
\end{array}$ \\
\hline Sim & $85 \%$ & $90 \%$ & $90 \%$ \\
\hline Não & $15 \%$ & $10 \%$ & $10 \%$ \\
\hline
\end{tabular}

Também nesta questão foi solicitado um comentário complementar. Apresentam-se duas destas justificativas:

Sim, até por ser uma tecnologia móvel poderá ser acessado em diversos lugares, onde há cobertura da operadora (Aluno 4 - Análise e Desenvolvimento de Sistemas).

Seria ótimo poder, em qualquer lugar, utilizar esses aparelhos para fins educativos e como futura professora estar sempre atualizada para melhorar a minha prática pedagógica (Aluno 10 - Licenciatura em Matemática).

Os alunos que não consideraram viável justificaram por razões pertinentes, já bem relatadas em estudos da área, tais como: tamanho da tela, preço do acesso à Internet e variedade de modelos de celular (o que poderia dificultar o acesso a certos arquivos).

Ressalta-se que nenhum dos participantes foi, previamente, orientado sobre o que seria m-learning. Isso torna as respostas ainda mais interessantes, uma vez que a maioria apresentou justificativas bem coerentes com as razões pelas quais a m-learning vem sendo defendida. Foi possível observar que, de maneira geral, os dados levantados, nos três cursos presenciais, indicaram a existência de um campo receptivo a ações educacionais utilizando dispositivos móveis, em particular, celulares.

\section{Considerações Finais}

A literatura sobre m-learning aponta limitações e vantagens da utilização de dispositivos móveis em educação. Tamanho de tela e de teclas e adaptação de aplicações desenvolvidas para Web são algumas dificuldades relatadas. Algumas sugestões têm sido propostas, tais como projeção da informação no ar, a partir da tela, e uso de teclado virtual. Entre as vantagens, são destacadas, em geral, interatividade, mobilidade, alcance de maior número de pessoas, prática de trabalho em equipes, aprendizagens em contextos reais, entre outros.

Com relação à Matemática, de forma geral, as tecnologias digitais abrem possibilidades, permitindo simulações, visualizações, experimentações, levantamento de hipóteses, entre outras ações. Com m-learning, adicionam-se possibilidades extras, como as mencionadas no parágrafo anterior. $\mathrm{O}$ uso, em dispositivos móveis, de aplicativos específicos para Matemática, ou mesmo, de recursos gerais, como SMS, fotografias, vídeos, entre outros, pode favorecer o processo de ensino e aprendizagem de Matemática, tornando-o mais acessível e mais próximo da realidade do aluno.

As potencialidades da m-learning têm sido consideradas nas diversas modalidades educacionais, indo além do foco inicial em educação a distância. Nesse sentido, pesquisas sobre m-learning e Matemática devem contemplar a educação a distância, a 
presencial e a semipresencial. Tais estudos podem favorecer não só a aprendizagem matemática, mas também a própria evolução da m-learning, pelos recursos desenvolvidos, identificação de formas de utilização e feedbacks obtidos.

Em termos de educação presencial, a pesquisa exploratória, promovida em três cursos do IF Fluminense, sinalizou uma boa receptividade quanto à utilização de dispositivos móveis para fins educacionais. Ressalta-se que, nos comentários dos participantes, foi possível identificar boa parte das vantagens e desvantagens de mlearning, apresentadas na literatura da área. Este fato indica uma coerência entre as concepções acadêmicas sobre o assunto e a visão destes jovens. A postura receptiva dos participantes da Licenciatura em Matemática sugere que dispositivos móveis podem ser recursos interessantes a serem trabalhados na formação inicial destes profissionais.

Notas de Texto

${ }^{1}<$ http://www.nokia.com/corporate-responsibility/society/mobile-technology-for-development/mobilelearning-for-mathematics>.

\section{Referências Bibliográficas}

BAYA'A, N.; DAHER, W. Students' perceptions of Mathematics learning using mobile phones. In: INTERNATIONAL CONFERENCE ON MOBILE AND COMPUTER AIDED LEARNING, 4., 2009, Amman, Jordan. Disponível em: $<$ http://users.qsm.ac.il/cellmath/Material/Conferences/IMCL2009/students_perceptions_of_ mathemat.pdf>. Acesso em: 20 set. 2009.

BOTZER, G.; YERUSHALMY, M. Mobile application for mobile learning. In: INTERNATIONAL CONFERENCE ON COGNITION AND EXPLORATORY LEARNING IN DIGITAL AGE (CELDA), 2007, Algrave, Portugal. Disponível em: <http://www.iadis.net/dl/final_uploads/200714C043.pdf>. Acesso em: 16 set. 2009.

BRASIL. Decreto $\mathbf{n}^{\circ} \mathbf{5 . 6 2 2}$, de 19 de dezembro de 2005. Disponível em: $<$ http://portal.mec.gov.br/seed/arquivos/pdf/dec_5622.pdf>. Acesso em: 18/09/09.

BROWN, R.; RYU, H; PARSONS, D. Mobile helper for university students: a design for a mobile learning environment. In: CONFERENCE ON COMPUTER-HUMAN INTERACTION-DESIGN: ACTIVITIES, ARTEFACTS AND ENVIRONMENTS, 18., 2006, Sydney, Australia. Proceedings. New York, USA: ACM, 2006, p. 297 - 300.

CASTELLS, M.; FERNANDEZ-ARDEVOL, M.; QIU, J. L.; SEY. A. The Mobile Communication Society: a across-cultural analysis of available evidence on the social uses of wireless communication technology (Relatório de pesquisa). In: INTERNATIONAL WORKSHOP ON WIRELESS COMMUNICATION POLICIES AND PROSPECTS: A GLOBAL PERSPECTIVE, HELD AT THE ANNENBERG SCHOOL FOR COMMUNICATION, 2004, Los Angeles, USA. Disponível em: $<$ http://arnic.info/workshop04/MCS.pdf>. Acesso em: 03 set. 2009.

COSTABILE, M. F.; DE ANGELI. A.; LANZILOTTI, R; ARDITO, C.; BUONO, P.; PEDERSON, T. Explore! Possibilities and Challenges of Mobile Learning. In: CONFERENCE ON HUMAN FACTORS IN COMPUTING SYSTEMS, 26., 2008, Florence, Italy. Proceedings. New York, USA: ACM, 2008. p. 145 - 154.

COSTELLO, F. The use of Flash Lite and web authoring tools in mobile learning course design. In: KEEGAN, D. (org.). Mobile learning: a practical guide (Leonardo da Vinci Programme of the European Commission). 2007. p. 79-91. Disponível em: $<$ http://www.ericsson.com/ericsson/corpinfo/programs/incorporating_mobile_learning_into _mainstream_education/>. Acesso em: 03 set. 2009.

DYE, A. Designing courseware for mobile devices. In: KEEGAN, D. (org.) Mobile learning: a practical guide (Leonardo da Vinci Programme of the European Commission). 2007. p. 51-56. Disponível em: 
$<\mathrm{http} / /$ www.ericsson.com/ericsson/corpinfo/programs/incorporating_mobile_learning_into _mainstream_education/>. Acesso em: 03 set. 2009.

FRANKLIN, T.; PENG, LI-W. Mobile math: math educators and students engage in mobile learning. Journal of Computing in Higher Education, Boston, EUA: Springer Boston. v. 20, n. 2. p. 69-80, 2008. DOI 10.1007/s12528-008-9005-0.

GEORGIEV, T.; GEORGIEVA, E.; SMRIKAROV, A. M-learning - a new stage of elearning. In: INTERNATIONAL CONFERENCE COMPUTER SYSTEMS AND TECHNOLOGIES, 5., 2004, Rousse, Bulgaria. Proceedings. New York, USA, ACM, 2004. p. $1-5$.

HERNÁNDEZ SAMPIERI, R.; FERNÁNDEZ COLLADO, C.; BAPTISTA LUCIO, P. Metodologia de la investigación. México: McGraw-Hill. 1998.

KEEGAN, D. (org.) Mobile learning: a practical guide (Leonardo da Vinci Programme of the European Commission). 2007. Disponível em: $<$ http://www.ericsson.com/ericsson/corpinfo/programs/incorporating_mobile_learning_into _mainstream_education/>. Acesso em: 03 set. 2009.

KHADDAGE, F.; LATTEMANN, C. Towards an ad-hoc mobile social learning network using mobile phones. In: INTERACTIVE COMPUTER AIDED LEARNING, 12., 2009, Villach, Austria. Proceedings. Villach, Austria: Fachhochschule Kärnten, 2009. p. 374 -380. MATH4MOBILE. Development. s.d. Disponível em: $<$ http://www.math4mobile.com/development >. Acesso em: 02 set. 2009.

MATOS, J. F. L. Matemática, Educação e Desenvolvimento Social - questionando mitos que sustentam opções atuais em desenvolvimento curricular em Matemática. In: ENCONTRO INTERNACIONAL EM HOMENAGEM A PAULO ABRANTES EDUCAÇÃO MATEMÁTICA: caminhos e encruzilhadas. Lisboa, 2005. Disponível em: $<$ http://www.educ.fc.ul.pt/docentes/jfmatos/comunicacoes.html>. Acesso em: 30 ago. 2009.

MEIRELLES, L. F. T.; TAROUCO, L. M. R. Framework para aprendizagem com mobilidade. In: SIMPÓSIO BRASILEIRO DE INFORMÁTICA NA EDUCAÇÃO, 16., 2005, Juiz de Fora, MG. Anais. Juiz de Fora, MG: SBIE, 2005.

MEISENBERGER, M. MLE - Mobile Learning Engine. Graz, Austria: FH JOANNEUM - University of Applied Sciences, 2004. Diploma Thesis.

MORAN, J. M. O que é educação a distância. 2002. Disponível em: $<$ http://www.eca.usp.br/prof/moran/dist.htm>. Acesso em: 18 set. 2009.

NOKIA. Mobile learning for Mathematics. 2009. Disponível em: $<$ http://www.nokia.com/corporate-responsibility/society/mobile-technology-for development/mobile-learning-for-mathematics>. Acesso em: 28 set. 2009.

QUINN, C. mLearning: mobile, wireless, in-your-pocket learning. 2000. Disponível em: <http://www.linezine.com/2.1/features/cqmmwiyp.htm>. Acesso em: 26 ago. 2009.

SÁ, M. de; CARRIÇO, L.; DUARTE, L.; REIS, T. Supporting the design of mobile interactive artefacts. In: GALLUD, J. A.; LOZANO, M. D. (Eds.). Advances in Engineering Software (designing, modelling and implementing interactive systems). v. 40, n.12, p.1279-1286, dez. 2009. Elsevier. DOI: <10.1016/j.advengsoft.2009.01.021>. In Press.

SCOPEL, M.; KRATZ, R. D. A; RHEINHEIMER, L.R.; PINTO, S. C. C. S. Pocket: um ambiente de ensino à distância usando handhelds na formação de comunidades virtuais espontâneas. In: SIMPÓSIO BRASILEIRO DE INFORMÁTICA NA EDUCAÇÃO, 15., 2004, Manaus, AM. Anais. Manaus, AM: SBIE, 2004.

TAROUCO, L. M. R.; FABRE, M. C. J. M.; GRANDO, A. R. S.; KONRATH, M. L. P. Objetos de aprendizagem para m-learning. In: SUCESU - CONGRESSO NACIONAL DE TECNOLOGIA DA INFORMAÇÃO E COMUNICAÇÃO, 2004, Florianópolis, SC 
Disponível em: <http://www.cinted.ufrgs.br/CESTA/objetosdeaprendizagem_sucesu.pdf>. Acesso em: 16 set. 2009.

WAINS, S. I.; MAHMOOD, W. Integrating m-learning with e-learning. In: CONFERENCE ON INFORMATION TECHNOLOGY EDUCATION, 9., 2008, Cincinnati, OH, USA. Proceedings. New York, USA, ACM, 2008. p. 31-38.

ZEILLER, M. Podcasting-based mobile learning in blended learning courses. In: INTERACTIVE COMPUTER AIDED LEARNING, 12., 2009, Villach, Austria. Proceedings. Villach, Austria: Fachhochschule Kärnten, 2009. p. 518 -527. 